\title{
Die audiovisuelle Übersetzung von Wortspielen am Beispiel der ecuadorianischen Webserie Enchufe.tv ${ }^{1}$
}

\author{
[The Audiovisual Translation of Wordplay Illustrated by the Example of the Ecuadorian Web \\ Series Enchufe.tv] \\ http://dx.doi.org/10.11606/1982-8837213547
}

\author{
Timur Stein ${ }^{2}$
}

\begin{abstract}
The ongoing internationalization of audiovisual media increases the need for new ways of overcoming linguistic barriers. In Germany, where the common technique has always been dubbing, subtitles are however becoming more and more popular. The present article will deal with the application possibilities of subtitles in audiovisual translation with reference to humoristic elements. The article will present two theoretical approaches to the translation of humour which will be then put into practice by using three sketches of the Ecuadorian web series Enchufe.tv as an example. All three sketches are based on wordplays, a manifestation of humour which is extremely difficult to reproduce in other languages. By this means, the article will show the strengths of the methods presented, but also will point out the need for their further adaption to the specific characteristics of audiovisual texts.
\end{abstract}

Keywords: audiovisual translation, subtitling, pragmalinguistic approaches, General Theory of Verbal Humour, wordplays

Zusammenfassung: Die voranschreitende Internationalisierung audiovisueller Medien zieht die Notwendigkeit der Überbrückung sprachlicher Barrieren nach sich. In Deutschland, traditionell ein Synchronisationsland, werden dazu immer häufiger Untertitel herangezogen. Der vorliegende Beitrag setzt sich mit den Anwendungsmöglichkeiten dieser Form des audiovisuellen Übersetzens in Bezug auf humoristische Elemente auseinander. Dabei werden zwei theoretische Ansätze zur Übertragung des Komischen vorgestellt, die anschließend anhand des praxisnahen Beispiels dreier Sketche der ecuadorianischen Webserie Enchufe.tv auf die Probe gestellt werden. Den drei Sketchen ist gemein, dass sie sich auf Wortspiele, also auf eine Erscheinungsform des Komischen stützen, die sich der Wiedergabe in einer anderen Sprache in außergewöhnlichem Maße widersetzt. Auf diese Weise werden einerseits die Stärken der vorgestellten Methoden aufgezeigt, andererseits wird aber auch die Notwendigkeit ihrer Ausweitung auf die besonderen Eigenschaften audiovisueller Texte deutlich.

\footnotetext{
${ }^{1}$ Der vorliegende Artikel bildet eine vertiefte Auseinandersetzung mit einem der Interessenschwerpunkte einer Abschlussarbeit im Fach Translation über die Möglichkeiten und Grenzen der Untertitelung des Komischen (s. STEIN, 2018). Mein besonderer Dank gilt Dr. phil. Marcel Vejmelka, Johannes Gutenberg-Universität Mainz, für seine fachliche Betreuung und seinen wertvollen Rat.

${ }^{2}$ Johannes Gutenberg-Universität Mainz, Fachbereich Translations-, Sprach- und Kulturwissenschaft (FTSK), An der Hochschule 2, 76726 Germersheim, Deutschland, E-Mail: timur.stein@yahoo.de.
}

\section{BY-NC}

Pandaemonium, São Paulo, v. 21, n. 35, set.-dez. 2018, p. 47-68 
Stein, T. - Die audiovisuelle Übersetzung von Wortspielen

Stichwörter: audiovisuelles Übersetzen, Untertitelung, pragmalinguistische Ansätze, General Theory of Verbal Humour, Wortspiele

\section{Einleitung}

Das Komische zu übersetzen, heißt in erster Linie, es in einen neuen kulturellen und sprachlichen Kontext einzubetten. Was die außerordentliche Herausforderung dieser translatorischen Aufgabe ausmacht, ist die Notwendigkeit, die komische Wirkung dem Rezipienten des Zieltextes nicht nur begreiflich, sondern insbesondere auch nachvollziehbar zu machen, denn nur so kann sie sich in ihrem ursprünglichen Sinne entfalten. Im Umkehrschluss lässt sich daher sagen, dass eine Übersetzung, die niemand als belustigend empfindet, ihrer kommunikativen Funktion in keiner Weise gerecht wird. Bedauerlicherweise stößt der Versuch, die komische Wirkung des Ausgangstextes adäquat wiederzugeben, in der Praxis oftmals auf vielerlei scheinbar unüberwindbare Hürden, die schon einmal dazu verleiten können, die Übersetzungsschwierigkeiten, die das Komische in sich birgt, denen der Poesie gleichzusetzen: „When it comes to translating humor, the operation proves to be as desperate as that of translating poetry“ (DIOT 1989: 84). Traditionell wird diese relative oder gar absolute Unübersetzbarkeit humoristischer Texte auf das Vorhandensein bzw. Zusammenwirken sprachund kulturspezifischer Eigenheiten des Ausgangstextes zurückgeführt (VANDAELE 2010). Für eine gelungene Übertragung verlangen sie dem Übersetzer oft interkulturelle Kompetenz, tiefgreifende Kenntnisse beider Sprachen und Kulturen sowie ein großes Maß an Kreativität ab.

Die beachtlichen Hürden, denen sich der Übersetzer humoristischer Texte gegenübersieht, offenbaren die Notwendigkeit methodischer Grundlagen zur Erleichterung und Qualitätssicherung des Arbeitsprozesses. Angesichts dessen, dass das Komische eine nur schwer fassbare Erscheinung darstellt, die von einer Vielzahl an sprachlichen und außersprachlichen Faktoren sowie rein subjektiver Wahrnehmung bedingt wird, ist es nicht verwunderlich, dass entsprechende Ansätze in der Fachliteratur nur spärlich gesät sind. Erschwerend kommt hinzu, dass die bis dato ausgearbeiteten Strategien im Arbeitsalltag professioneller Sprachmittler kaum Anwendung finden. Das Ziel der vorliegenden Arbeit ist es daher, einen Beitrag zur Verknüpfung von Theorie und Praxis zu leisten. In diesem Sinne werden zum einen Hickeys (1999) pragmalinguistische Ansätze und zum anderen die General Theory of Verbal Humour (GTVH) nach Attardo (2002) vorgestellt. Erstere dienen der Analyse humoristischer Texte und der Ausarbeitung geeigneter Übersetzungsvorschläge unter 
Stein, T. - Die audiovisuelle Übersetzung von Wortspielen

Berücksichtigung der perlokutionären Funktion des Ausgangstextes, während die GTVH ein umfassendes Instrumentarium zur Evaluierung des Endprodukts der Übertragung komischer Elemente bietet. Beide Methoden werden anhand eigens für diesen Zweck erstellter deutscher Untertitelungen der ecuadorianischen humoristischen Webserie Enchufe.tv auf ihre Funktionsweise in einer praxisnahen Situation hin überprüft. Das Kriterium für die Auswahl der Videos war dabei, dass sich die Auflösung der Witzeinheit, die sie beinhalten, auf Wortspiele stützt. Der Grund dafür sind die beachtlichen Schwierigkeiten, welche die Übersetzung von Wortspielen, die sich oftmals in der jeweiligen Sprache vorhandene phonetische Zufälle zunutze machen, mit sich bringt. Da sich der Translationsprozess durch die Einschränkungen audiovisueller Texte, die unter anderem in Form von unterschiedlichen Zeichenmodalitäten in Erscheinung treten, zusätzlich beeinträchtigt sieht, bestehen ideale Bedingungen dafür, das Potential der hier behandelten Übersetzungs- und Evaluierungsstrategien auszuschöpfen bzw. auf die Probe zu stellen.

Bevor eine vertiefte Auseinandersetzung mit den angefertigten Übersetzungen stattfindet, erfolgt eine kurze Einführung in die sogenannten Humour Studies. Die in diesem interdisziplinären Forschungszweig gewonnenen Erkenntnisse bilden eine wichtige Grundlage für die theoretische Annäherung an das Gebiet des Komischen.

\section{Die Humour Studies}

Nicht nur die Translationswissenschaft befasst sich mit dem Komischen, auch in anderen Disziplinen wie etwa der Soziologie, Physiologie und Medizin findet schon seit Langem eine wissenschaftliche Auseinandersetzung mit diesem Phänomen statt. Wie jedoch die Translationswissenschaftlerin Santana López beobachtet, wird die interdisziplinäre Beschaffenheit des Komischen „oft übersehen bzw. unterschätzt“ (SANTANA LÓPEZ 2006: 14). Die Humour Studies wirken dem entgegen, indem sie Vertreter unterschiedlicher Forschungsrichtungen vereinen und ihren gemeinsamen Untersuchungsgegenstand, das Komische, aus unterschiedlichen Blickwinkeln zu durchleuchten suchen.

Interessant ist für unsere Belange die Tatsache, dass sich in den Humour Studies zwei Interpretationslinien durchsetzen konnten, welche die Tatsache, dass etwas als belustigend aufgefasst wird, auf unterschiedliche Ursachen zurückführen. Auf der einen Seite steht die sogenannte Superioritätstheorie. Diese stützt sich auf einen von Platon begründeten 
Stein, T. - Die audiovisuelle Übersetzung von Wortspielen

philosophischen Diskurs. In Philebos stellt er das edle Vergnügen der Tragödie dem der Komödie gegenüber. Letztere geht als geringwertig aus diesem Vergleich, da sie ,auf Bosheit und Neid, auf dem Genuß [sic] des Übels, das einem anderen zustößt“, beruhe (SCHROEDER 2002: 25). Eine ähnliche Haltung gegenüber dem Lachen vertritt auch Hobbes, der es in sein nicht allzu positiv geprägtes Welt- und Menschenbild einbettet. Für ihn stellt es, so Schroeder (2002: 32), ,eine der verwerflichsten Eigenschaften des Menschen“ dar. Tatsächlich betrachtet Hobbes das Lachen lediglich als Instrument zur Steigerung der eigenen Stellung auf fremde Kosten:

Bei plötzlicher Freude über ein Wort, eine Tat, einen Gedanken, die das eigene Ansehen erhöhen, das fremde mindern, werden [...] die Lebensgeister emporgetrieben, und dies ist die Empfindung des Lachens (HOBBES 1966: 33).

Die Selbstaffirmation durch Auslachen ist ein grundlegender Bestandteil der Superioritätstheorie. So bedienen sich beispielsweise diejenigen Witze dieser Art von Komik, die Individuen oder gar Personengruppen zu ihrer Zielscheibe erklären.

Im Gegensatz dazu steht hinter der Inkongruenztheorie eine philosophische Tradition, die das Lachen nicht verurteilt, sondern vielmehr als rein menschlich zu beschreiben sucht. So betrachtet es Kant als ein Produkt getäuschter rationaler Erwartung:

Es muß in allem, was ein lebhaftes erschütterndes Lachen erregen soll, etwas Widersinniges seyn (woran also der Verstand an sich kein Wohlgefallen finden kann). Das Lachen ist ein Affekt aus der plötzlichen Verwandlung einer gespannten Erwartung in nichts (KANT 1792: 222).

Auch für Schopenhauer ist in Die Welt als Wille und Vorstellung das Überraschungsmoment, also die plötzlich eintretende Einsicht eines Irrtums entscheidend. Dazu müsse eine reale Situation einer falschen Kategorie zugeordnet werden, denn die Wahrnehmung der Inkongruenz zwischen Wirklichkeit und Gedachtem bereite Freude. Diese resultiere insbesondere aus der Erkenntnis der Unvollkommenheit des Verstandes: „Diese strenge, unermüdliche, überlästige Hofmeisterin Vernunft jetzt ein Mal der Unzulänglichkeit überführt zu sehen, muss uns daher ergötzlich seyn“"(SCHOPENHAUER 1916: 122).

In den Humour Studies herrscht heute Konsens über den Dualismus zwischen Superiorität (Selbstaffirmation durch Erniedrigung Dritter) und Inkongruenz (überraschende Einsicht der Unzulänglichkeit des Verstandes) und ihre Rolle für die Erzeugung komischer Wirkung. Dabei wird nicht außer Acht gelassen, dass die beiden auf den ersten Blick so 
Stein, T. - Die audiovisuelle Übersetzung von Wortspielen

unterschiedlichen Kategorien durchaus gemeinsame Schnittpunkte haben können: ,incongruity and superiority do not represent incompatible views of humor but are strongly interwoven“ (VANDAELE 2002: 221). Nichtsdestotrotz sei an dieser Stelle darauf hingewiesen, dass für die vorliegende Arbeit in erster Linie inkongruenzbasierte Komik von Interesse ist. Der Grund dafür sind die besonderen Merkmale von Wortspielen, die folgendermaßen definiert werden können:

Wordplay is the general name for the various textual phenomena in which structural features of the language(s) used are exploited in order to bring about a communicatively significant confrontation of two (or more) linguistic structures with more or less similar forms and more or less different meanings (DELABASTITA 1996: 128).

Es steht also fest, dass Wortspiele sich die Homophonie und/oder Polysemie einzelner sprachlicher Einheiten zunutze machen, indem sie sie einander direkt gegenüberstellen. Wird der Rezipient eines Witzes dadurch in die Irre geführt, erfreut er sich dieser Einsicht. Dies lässt sich an Beispielwitz (1) gut veranschaulichen:

(1) Treffen sich eines Tages zwei Fische. Sagt der eine zum anderen „Hi!“, woraufhin dieser erschrocken herumfährt und ruft „Wo?“

Der Leser dieses Witzes erkennt $h i$ als Grußwort und genauso ergeht es dem Hörer, der angesichts der Begegnung beider Fische zur selben Interpretation neigt. Durch die Erkenntnis der Homophonie der Wörter hi und Hai muss er sich seinen Irrtum jedoch eingestehen. Es kann somit festgestellt werden, dass sich dieses Wortspiel inkongruenter Komik bedient.

\section{Hickeys pragmalinguistische Übersetzungsstrategie}

Wie eingangs angekündigt, wenden wir uns nun Hickeys (1999) pragmalinguistischen Ansätzen zu. Auf ihnen aufbauend entwickelt Hickey eine simple und praxisorientierte Methode für die Übertragung komischer Wirkung. Ihre Anwendungsmöglichkeiten und Reichweite demonstriert er an einer Vielzahl von Beispielwitzen, die teils komplexe kulturelle und sprachliche Konstellationen aufweisen. Eines seiner Hauptanliegen ist es dabei, deutlich zu machen, welche Bedeutung einer eingehenden Ausgangstextanalyse zukommt. Aus Hickeys Ausführungen geht hervor, dass nur eine solche Vorgehensweise als Garant für einen erfolgreichen Umgang mit dem Komischen angesehen werden kann, das, wie er zugibt, der Übertragung in andere Sprachen mit allen Mitteln widerstrebt: „Es sabido que el humor viaja 
Stein, T. - Die audiovisuelle Übersetzung von Wortspielen

mal, que suele marchitarse en el trayecto más corto, llegando deshecho, si no muerto, a su destino, al trasladarse de un idioma a otro“ (HICKEY 1999).

Als Antwort auf die fragwürdige Übersetzbarkeit komischer Elemente sehen viele Übersetzer Hickey (1999) zufolge nicht selten den einzigen Ausweg darin, dem Rezipienten die ausgangssprachliche Komik anhand von Erläuterungen in der Zielsprache näherzubringen bzw. in ihrer Fülle verständlich zu machen. Es versteht sich von selbst, dass eine solche Vorgehensweise im audiovisuellen Übersetzen, das aufgrund seiner räumlichen und zeitlichen Einschränkungen keinen Platz für Kommentare bietet, nicht angewandt werden kann. Hinzu kommt die Tatsache, dass man auf diese Weise Gefahr laufen würde, die belustigende Wirkung gänzlich aufzulösen, denn bekanntermaßen ist das Erklären eines Witzes selten dazu geeignet, dem Zuhörer auch nur ein Schmunzeln zu entlocken.

Es steht fest, dass die zuvor beschriebene Vorgehensweise zumindest im audiovisuellen Bereich keine ernstzunehmende Option darstellt. ${ }^{3}$ Nun stellt sich die Frage, welcher Weg zu beschreiten ist, wenn eine stark am Original orientierte Wiedergabe der Komik den ursprünglichen Effekt nicht zu erzielen vermag. Als hilfreich erweisen sich hierbei die Erkenntnisse zur Gleichwertigkeit von Original und Translat, zu denen Reiss (2000) gelangt. So hebt sie unter anderem hervor, dass ganzheitliche Textäquivalenz vorliegen kann, ohne dass es dafür der Äquivalenz auf Segmentebene bedürfe. Diese Überzeugung teilt Hickey (1999) und führt sie mit Blick auf das Komische weiter aus. Seiner Ansicht nach sind nach einer vertieften Auseinandersetzung mit dem ausgangssprachlichen Witz die sprachlichen und pragmatischen Elemente zu eruieren, die für die Entfaltung der komischen Wirkung essentiell sind, während inessentielle Elemente bedenkenlos verändert werden könnten. Um das zu gewährleisten, bedürfe es in erster Linie einer pragmalinguistischen Analyse. Auf diese Weise soll festgehalten werden, welche perlokutionäre Wirkung zu erzeugen ist und welche Reaktion seitens des Lesers damit einherzugehen hat. In anderen Worten wird also versucht, dem Übersetzer humoristischer Texte etwas geradezu Selbstverständliches nahezubringen: Ein Witz kann nur dann erfolgreich in einer anderen Sprache und kulturellen Sphäre reproduziert werden, wenn man versteht, was genau ihn als solchen ausmacht.

Diese Vorgehensweise verhilft Hickey (1999) zufolge auch zu einem angemessenen Umgang mit Wortspielen, die aufgrund ihrer direkten Bindung an sprachspezifische Merkmale

\footnotetext{
${ }^{3}$ Im Falle einer Übersetzung, die lediglich zu Studienzwecken angefertigt wird, wäre dies eine durchaus denkbare Methode. Allerdings kommt dies aus den oben genannten Gründen bei audiovisuellen Texten nicht infrage.
} 
Stein, T. - Die audiovisuelle Übersetzung von Wortspielen

eine außergewöhnliche Herausforderung darzustellen pflegen. Dazu sei es oft erforderlich, sich auf semantischer Ebene vom ursprünglichen Witz zu entfernen. Dass es sich beim Endergebnis nicht mehr um denselben Witz handelt, wird dabei nicht als negativ aufgefasst:

[...] una vez analizado el rasgo lingüístico que crea el humor, ese rasgo puede dar de sí para muchos chistes gemelos o primos hermanos, eligiendo el traductor el revestimiento semántico que más le convenga (HICKEY 1999).

Kehren wir nun noch einmal zu Beispielwitz (1) zurück, um eine bessere Vorstellung davon zu entwickeln, wie ein solcher Übersetzungsprozess aussehen kann. Hier wird schnell klar, dass die falsche Deutung eines Begriffs, die mittels zweier Homophone herbeigeführt wird, für die komische Wirkung im Sinne der Inkongruenztheorie verantwortlich ist. Der Austausch der semantischen Struktur des Originals unter Erhalt der sprachlichen Elemente, die den Rezipienten in die Irre führen, kann auch in deutscher Sprache zu einer Vielzahl von Parallelwitzen führen, so etwa dem folgenden:

(2) Treffen sich zwei Schafe. Sagt das eine „Mäh“, worauf das andere erzürnt ruft „Mäh doch selber!“

An diesem Beispiel ist gut zu sehen, dass die Essenz des ursprünglichen Witzes trotz eines anderen semantischen Aufbaus beibehalten werden konnte. Der genaue Kontext, in dem sich die jeweilige Handlung abspielt, rückt in den Hintergrund. Somit ist es zweitrangig, ob der Dialog auf dem Meeresgrund oder auf der Wiese stattfindet. Was zählt ist einzig und allein die Täuschung des menschlichen Verstandes, die durch die Homophonie des Begriffes mäh (Tierlaut bzw. Imperativ der 2. Person Singular von mähen) gewährleistet wird, denn in ihr verbirgt sich dieselbe perlokutionäre Wirkung, die auch Variante (1) ausmacht.

Bei der Übersetzung findet dieses Prozedere in gleicher Weise Anwendung, muss unter Umständen jedoch aufgrund der Unterschiede zwischen Ausgangs- und Zielsprache unter erschwerten Bedingungen geschehen. ${ }^{4}$ Angenehmerweise trifft dies zumindest im Sprachenpaar Deutsch-Spanisch nicht auf Beispielwitz (1) zu, denn er lässt sich ohne größere Schwierigkeiten ins Spanische übersetzen:

(3) Un día de mar tranquilo, dos surfistas aburridos se encuentran en la playa. El uno dice “¡Hola!” a lo que el otro, sorprendido, se da la vuelta y exclama “¿Dónde?”

\footnotetext{
${ }^{4}$ Man denke nur an ein Wortspiel, das auf einem Kompositum gründet und in eine Sprache ohne Kompositabildung übertragen werden soll, so etwa im folgenden Witz: „Was sagt ein großer Stift zu einem kleinen Stift? Wachsmalstift!“, <https://www.uni-marburg.de/fb09/igs/mitarbeiter/wiese/linguistenwitze>.
} 
Stein, T. - Die audiovisuelle Übersetzung von Wortspielen

Beispielübersetzung (3) versucht gemäß Hickeys (1999) Vorstellungen, die perlokutionäre Wirkung des Ausgangstextes zu reproduzieren und bedient sich dazu adäquater sprachlicher Mittel, nämlich der Homophone hola und ola. Auch in diesem Fall führt eine Begrüßung zu einem Missverständnis und zu einer gleichfalls unerwarteten Reaktion seitens eines der Gesprächspartner. Die Entfaltung der komischen Wirkung wird dadurch, dass sich der Dialog nicht zwischen zwei Tieren abspielt, kaum beeinträchtigt. ${ }^{5}$ Es würde jedoch anders aussehen, wenn sie in einen größeren semantischen Kontext eingebunden wäre, da willkürliches Austauschen zum Verlust jeglicher Komik führen würde. Dieser Umstand wird von Hickey (1999) wohlgemerkt bedacht: „Naturalmente, en aquellos casos en los que la semántica sea relevante, habrá que respetarla".

Wie man erkennen kann, ist der soeben vorgestellte Ansatz stark praxisorientiert und sucht die Aufgabe des Übersetzers humoristischer Texte zu erleichtern. Gleichzeitig kann nicht darüber hinweggesehen werden, dass der auf diese Weise übertragene Witz nicht mehr ganz dem Original entspricht. Hickey (1999) verweist diesbezüglich darauf, dass es sich dabei um keine Übersetzung im engeren Sinne, sondern um Anpassung humoristischer Texte an die Zielkultur bzw. -sprache handele. Wie man jedoch an folgendem Kommentar zu einer Passage aus Burkhart Kroebers deutscher Übersetzung von Umberto Ecos Der Name der Rose, die das Original womöglich an Spitzfindigkeit übertrifft, sehen kann, befinden es nicht alle Translationstheoretiker für angebracht, die ursprüngliche Komik zu modifizieren: ,should the translator be allowed to make us laugh at his own ideas rather than at those of the author? We do not think so“" (STACKELBERG 1988: 13). Eine solche Haltung, welche die Unantastbarkeit des Originals in den Vordergrund rückt, erweist sich allerdings meist als praxisfern.

\section{Die General Theory of Verbal Humour}

Nach der Vorstellung einer konkreten Translationsstrategie kommen wir nun wie angekündigt zur General Theory of Verbal Humour (GTVH). Hierbei handelt es sich um eine 1991 von Attardo und Raskin eingeführte Theorie, die es ermöglicht, das Endprodukt der Übersetzung

\footnotetext{
${ }^{5}$ Natürlich hat allein schon die Tatsache, dass ein Dialog zwischen zwei Tieren stattfindet, etwas Belustigendes an sich, werden doch zwei sich ausschließende Kategorien verknüpft, nämlich, tierisches Dasein“ und ,menschliches Sprachvermögen‘. Allerdings ist für die Entfaltung der komischen Wirkung in Beispielwitz (1) nicht so sehr dieser Umstand, sondern viel mehr das Wortspiel zwischen hi und Hai von Belang, das bei Fischen eindeutig besser aufgehoben ist als bei Menschen.
} 
eines humoristischen Textes auszuwerten. Um das gewährleisten zu können, wurde ein Konzept entwickelt, das die Grundzüge der komischen Wirkung in der zu übertragenden Witzeinheit analysiert und beurteilt, ob sie sich durch den Translationsprozess zu weit vom Original wegbewegt hat. Auf den ersten Blick erinnert das an Stackelbergs (1988) Sorge, der Übersetzer würde den ursprünglichen Witz einfach austauschen. Allerdings weist die GTVH eine weitaus größere Toleranzspanne auf. So betont Attardo $(2002,184)$ ausdrücklich, dass der Anspruch auf absolute Übersetzung im Gebiet des Komischen utopisch sei. Folglich könnte über kleinere Abweichungen hinweggesehen werden.

Das Herzstück der GTVH ist die Annahme, dass sich jede Witzeinheit aus maximal sechs Parametern zusammensetzt, die auch als Knowledge Resources bezeichnet werden können und sich, wie später noch genauer erklärt wird, hierarchisch anordnen lassen. Nachfolgend werden sie kurz nach Attardo (2002) zusammengefasst. Daraufhin wird erläutert, in welcher Form sie in der GTVH Anwendung finden.

In der hierarchischen Abfolge steht für Attardo (2002) der Parameter Language (LA) an unterster Stelle. Hierbei handelt es sich um das sprachliche Material, das bei der Verbalisierung des jeweiligen Witzes Verwendung findet. Für LA ist die Möglichkeit des Paraphrasierens bezeichnend, da die meisten Witze umformuliert werden können, ohne dass ihre Wirkung durch diesen Prozess verloren ginge.

An nächster Stelle steht der Parameter Narrative Strategy (NS). Darunter ist die erzählerische Organisation des Witzes zu verstehen, der beispielsweise als ein Rätsel oder in Dialogform gestaltet werden kann. Wie im weiteren Verlauf der vorliegenden Arbeit zu sehen sein wird, hat der Übersetzer audiovisuellen Materials im Normalfall keinerlei Einfluss auf diesen Parameter. Das heißt also, dass NS im Gegensatz zu LA nicht modifiziert werden kann.

Die dritte Knowledge Resource wird als Target (TA) bezeichnet. Ein Witz enthält TA nur dann, wenn er ein Individuum oder eine Personengruppe unter Einbezug dazugehöriger Stereotype lächerlich macht, sich in anderen Worten also superioritätsbasierter Komik bedient. Da dies bei Weitem nicht immer der Fall ist, ist TA Attardo (2002) zufolge als ein optionaler Parameter zu betrachten.

Als Situation (SI) wird die situative Einbettung eines Witzes bezeichnet. Sie beinhaltet Informationen zu allen Gegenständen und Figuren, die für seine Auflösung relevant sind. Im Mittelpunkt steht dabei die von den Figuren ausgeführte Handlung: ,the activity constitutes the central element of the situation, which also includes, of course, the participants, objects, 
Stein, T. - Die audiovisuelle Übersetzung von Wortspielen

instruments, etc.“ (ATTARDO; RASKIN 1991: 303). Ähnlich wie bei NS hat der Übersetzer audiovisuellen Materials keinen Einfluss auf SI, da dieser Parameter bereits visuell vorgegeben ist. Dies wird später an der Besprechung der Untertitelung der Videos Terapia de Angustia und Pollo al Vino sehr gut zu sehen sein.

Unter Logical Mechanism (LM) verbirgt sich ein Parameter, der für die Auflösung des Witzes verantwortlich ist. Er nimmt innerhalb der GTVH eine entscheidende Position ein, da er die Entfaltung der komischen Wirkung überhaupt erst ermöglicht. Dazu muss LM es bewerkstelligen, die aufgebaute Inkongruenz aufzulösen. In unserem Witz (1) geschieht das beispielsweise anhand der Homophonie zweier Begriffe. Die Übersetzung (3) schafft es ihrerseits, die Auflösung des Witzes in gleicher Weise zu gestalten und behält dadurch den ursprünglichen LM bei. Interessanterweise weist Attardo (2002) zusätzlich darauf hin, dass es sich auch hierbei um einen optionalen Parameter handelt, da etwa absurde Witze keine Auflösung besitzen.

An oberster Stelle steht Script Opposition (SO). Um die Funktionsweise dieses Parameters nachvollziehen zu können, muss zuallererst geklärt werden, was sich unter dem Begriff script verbirgt. Schauen wir uns dazu folgende Definition an:

A script or frame is an organized complex of information about something, in the broadest sense: an object (real or imaginary), an event, an action, a quality, etc. It is a cognitive structure internalized by the speaker which provides the speaker with information on how the world is organized, including how one acts in it (ATTARDO 2002: 121).

Bei Skripten handelt es sich also um kognitive Strukturen, die mit Informationen zur uns umgebenden Realität angereichert sind und dabei helfen, sie besser zu verstehen. Attardo (2002) zufolge müssen zwei Bedingungen erfüllt sein, damit ein Witz als solcher aufgefasst wird: Zum einen muss er zwei denkbare Skripte aufweisen und zum anderen bedarf es einer partiellen oder absoluten Überschneidung dieser. An Beispielwitz (1) lässt sich sehr gut verdeutlichen, was genau damit gemeint ist. Auch wenn hier im ersten Moment der Eindruck entsteht, es läge nur das Skript ,Begrüßung‘ vor, ist das Skript ,Warnruf‘ zumindest in dem Abschnitt „Treffen sich eines Tages zwei Fische. Sagt der eine zum anderen ,Hi!““ damit deckungsgleich. Dabei ist es wohlgemerkt irrelevant, dass der Leser dieses Witzes hi eindeutig als Grußwort identifiziert, denn eigentlich geht es ausschließlich um die angenommene akustische Vernehmung seitens des zweiten Fisches. Beide Skripte überschneiden sich nicht nur, sie sind einander auch entgegengesetzt, da sie völlig unterschiedliche Vorstellungen vermitteln.

Pandaemonium, São Paulo, v. 21, n. 35, set.-dez. 2018, p. 47-68 
Stein, T. - Die audiovisuelle Übersetzung von Wortspielen

Wie bereits erwähnt wurde, stützt sich die GTVH auf diese sechs Parameter. Mit ihrer Hilfe wird beurteilt, ob und - wenn ja - bis zu welchem Grad die Übersetzung des jeweiligen Witzes vom Original abweicht. Um das zu bewerkstelligen, nimmt Attardo (2002) eine Hierarchisierung der Knowledge Resources vor. Wie in Abbildung $1 \mathrm{zu}$ sehen ist, nimmt SO dabei den höchsten Rang ein. Das bedeutet nichts anderes, als dass eine Abweichung hier als gravierender aufgefasst wird als in einem niedrigeren Parameter. So würden ein Witz und seine zielsprachliche Wiedergabe, die sich lediglich in LA und/oder NS unterscheiden, im Gegensatz dazu als relativ ähnlich empfunden.

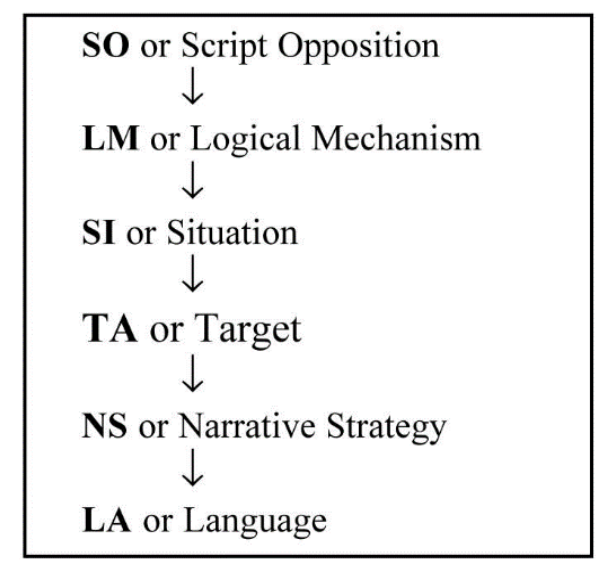

ABBILDUNG 1

Hierarchische Anordnung der Knowledge Resources

(Quelle: ATTARDO 2002: 183)

Das hierarchische Parameter-Modell der GTVH wird in der soeben dargestellten Form bei nahezu allen Ausdrucksformen des Komischen angewendet. Die einzige Ausnahme bildet das Übersetzen von Wortspielen. Der dazu verwendete logische Mechanismus, den Attardo (2002) als cratylism ${ }^{6}$ bezeichnet, wirkt sich stark einschränkend auf das sprachliche Material (LA) aus: ,it limits the options of the Language Knowledge Resource to any of the options which fulfil the requirements of the Logical Mechanism“ (ATTARDO 2002: 189). Im Umkehrschluss bedeutet das aber auch, dass LA bei Wortspielen einen weitaus höheren Rang einnimmt, da es ohne eine präzise Abstimmung dieses Parameters zu keiner Auflösung der Witzeinheit kommen kann.

\footnotetext{
${ }^{6}$ Cratylism ist als der logische Mechanismus zu verstehen, der für die Auflösung von Wortspielen verantwortlich ist. Eine detaillierte Übersicht aller bekannten logischen Mechanismen findet sich in Attardo; Hempelmann; Maio (2002).
} 
Stein, T. - Die audiovisuelle Übersetzung von Wortspielen

Zur Verdeutlichung der Funktionsweise der GTVH werden der Beispielwitz (1) und seine spanische Übersetzung (3) herangezogen:

(1) Treffen sich eines Tages zwei Fische. Sagt der eine zum anderen „Hi!“, woraufhin dieser erschrocken herumfährt und ruft „Wo?““

(3) Un día de mar tranquilo, dos surfistas aburridos se encuentran en la playa. El uno dice "¡Hola!" a lo que el otro, sorprendido, se da la vuelta y exclama “¿Dónde?”

Das sprachliche Material in (3) entspricht in seinen Grundzügen dem aus (1), da zur Herstellung des Wortspiels ebenfalls ein homophones Wortpaar verwendet wird. ${ }^{7}$ Das Gleiche gilt für NS, denn beide Witze sind dialogisch aufgebaut. TA betreffend ist lediglich anzumerken, dass es sich um keine superioritätsbasierte Komik handelt und dieser optionale Parameter hier daher entfällt. Eine Abweichung vom ausgangssprachlichen Witz findet sich in SI, da die Suche nach einem parallelen Wortspiel eine andere Situierung erforderlich machte. Im Gegensatz dazu entsprechen jedoch LM (cratylism) und SO (Opposition der Skripte ,Begrüßung ${ }^{6}$ und ,Lenken der Aufmerksamkeit auf einen bestimmten Sachverhalt') dem Original.

Wir stellen fest, dass sich die Varianten (1) und (3) nur in Bezug auf SI voneinander wegbewegen. Da es sich jedoch um einen relativ hohen Parameter handelt, muss geschlussfolgert werden, dass beide Witze nicht als identisch aufgefasst werden können. Nichtsdestotrotz bleibt die Essenz des Wortspiels erhalten, weshalb die Übersetzung gemäß der GTVH als zufriedenstellend zu bewerten ist.

\section{Anwendung der vorgestellten Methoden auf den Korpus}

Kommen wir nun zur Auswertung der deutschen Untertitelung dreier Sketche der ecuadorianischen Webserie Enchufe.tv. Sie alle enthalten Wortspiele und an ihrem Beispiel gilt es nun, die Funktionalität der zuvor vorgestellten Übersetzungs- und Evaluierungsmethoden auf die Probe zu stellen.

Es kam soeben zur Sprache, dass das Übersetzen von Wortspielen mit einer starken Einschränkung in Bezug auf das sprachliche Material verbunden ist. Abhängig von den

\footnotetext{
${ }^{7}$ Da die komische Wirkung des Ausgangstextes auf dem Zusammenspiel zweier Homophone gründet, würde die Verwendung von Homonymen im Zieltext eine Abweichung vom ursprünglichen sprachlichen Material bedeuten, die jedoch so geringfügig wäre, dass sie meines Erachtens bedenkenlos hingenommen werden könnte.
} 
Stein, T. - Die audiovisuelle Übersetzung von Wortspielen

jeweiligen Rahmenbedingungen kann diese jedoch auch recht geringfügig ausfallen. Ein Beispiel hierfür ist Teléfono Dañado ${ }^{8}$ (s. Tabelle 1). In diesem Sketch spielen vier Personen ,Stille Post‘. Der letzte Spieler, also derjenige, der verraten muss, welche Nachricht bei ihm angelangt ist, erschießt den jeweils ersten Spieler, da die ursprüngliche Botschaft in verfälschter Form ankommt und als Beleidigung wahrgenommen wird. So wird der Satz „El maní es una fruta“ (Erdnüsse sind Obst) zu ¿Que mi mami es una puta? (Meine Mama ist eine Hure?).

TABELLE 1

Teléfono Dañado

\begin{tabular}{llll} 
Zeitcode & Sprecher & Spanisch & Deutsch \\
\hline 00:22,140 $\rightarrow$ & Spieler 1 & El maní es una fruta. & Das Futter ist für die Stute. \\
00:24,200 & & & \\
\hline 00:38,180 $\rightarrow$ & Bewaffneter & ¿Que mi mami es una puta? & Meine Mutter ist eine Nutte? \\
00:39,800 & & & \\
\hline 00:53,980 $\rightarrow$ & Spieler 2 & La papa va bien con alverja. & Mein Kater trägt einen \\
00:56,340 & & & Blumenkranz. \\
\hline 00:56,600 $\rightarrow$ & Spielerin 3 & \multirow{2}{}{ ¿No! } & Oh nein! \\
00:57,070 & & &
\end{tabular}

Die Untertitelung dieses Videos ist als unproblematisch $\mathrm{zu}$ bewerten. Die zu übermittelnden Botschaften sind visuell nicht verfestigt. Somit besteht für den Übersetzer keine semantische Bindung und er verfügt dadurch über einen gewaltigen Handlungsspielraum.

Bei der Übertragung erfolge gemäß Hickeys (1999) Ansätzen an allererster Stelle eine Analyse der perlokutionären Wirkung des Sketches. Dabei konnte festgestellt werden, dass sie sich in großem Maße auf den Satz „La papa va bien con alverja“ (Kartoffeln passen gut zu Erbsen) stützt, der wie der erste ebenfalls hohes Potential birgt, missverstanden zu werden. Der verzweifelte Ausruf der Spielerin, die diese Botschaft an den Bewaffneten zu übermitteln hat, bestätigt die Aussicht auf einen fatalen Ausgang der Spielrunde. Da der Sketch jedoch an dieser Stelle endet, bleibt es dem Zuschauer überlassen, sich zu überlegen, wie die Endbotschaft lauten könnte. Ebendiese Wirkung gilt es in der deutschen Untertitelung zu reproduzieren. Die einzige Vorgabe ist dabei, dass der zielsprachliche Satz phonetisch so beschaffen sein muss, dass er bei einer mangelhaften Übermittlung als Beleidigung verstanden werden kann. Dies ermöglicht es

\footnotetext{
${ }^{8}<$ https://www.youtube.com/watch?v=Fpfk-Afcol0>

Pandaemonium, São Paulo, v. 21, n. 35, set.-dez. 2018, p. 47-68
} 
Stein, T. - Die audiovisuelle Übersetzung von Wortspielen

dem Übersetzer, seiner Fantasie bis zu einem gewissen Grad freien Lauf zu lassen und aus einer Vielzahl möglicher Parallelwitze denjenigen auszusuchen, der seinen Vorstellungen am besten entspricht. So hat die Aussage „Das Futter ist für die Stute“ zwar auf den ersten Blick nichts mit dem Ausgangstext gemein, resultiert aber in einer gleichwertigen Beleidigung: „Meine Mutter ist eine Nutte?“. Bei der zweiten Botschaft, welche unter anderem die Deutung als „Tú papá es un cara de verga/careverga“ (Dein Papa ist ein Schwanzgesicht) impliziert, wurde versucht, die Elemente ,papá (Papa) und das vulgäre ,verga (Schwanz) beizubehalten. Die Wiedergabe mit „Mein Kater trägt einen Blumenkranz“ bewahrt die perlokutionäre Wirkung des Originals, indem der Rezipient der Untertitelung dazu aufgefordert wird, zu überlegen, wie eine verfälschte Übermittlung dieses Satzes aussehen könnte. ${ }^{9}$ Es ist an letzter Stelle lediglich deutlich zu machen, dass die soeben vorgestellte Übersetzung nur eine von vielen Varianten darstellt, die etwa „Mein Kater hat einen gestreiften Schwanz“, „Mein Kater hat einen dicken Wanst“ oder auch „Der Marder isst sein Lieblingsgericht“ lauten könnten.

Hickeys (1999) Methode befolgend, gestaltete sich die Übersetzung von Teléfono Dañado ohne größere Schwierigkeiten. Nun stellt sich jedoch die Frage, ob es sich dabei noch um denselben Witz handelt. Nachfolgend wird ihr anhand der GTVH nachgegangen. In Bezug auf die Knowledge Resources fällt zuallererst auf, dass TA nicht vorhanden ist, während NS (Botschaft vs. Ergebnis) und SI (Gruppenspiel) durch das audiovisuelle Material vorgegeben sind. Die Anforderungen des herzustellenden bzw. anzudeutenden Wortspiels erlegen zwar feste sprachliche Vorgaben auf, doch konnte ihnen mit etwas Einfallsreichtum problemlos entsprochen werden. Dass sich LA im Zieltext dabei auf den ersten Blick stark vom Ausgangstext unterscheidet, hat aber wohlgemerkt keine Auswirkungen auf die Wahrnehmung des Witzes, denn die neu geformten Satzpaare gewährleisten eine Auflösung (LM) nach Vorbild des Originals. Zuallerletzt ist darauf hinzuweisen, dass in der Untertitelung auch SO vorhanden ist. Die Skriptopposition äußert sich in Form zweier einander entgegengesetzter und sich partiell überschneidender Skripte. Das erste Skript stellt die Möglichkeit dar, dass Spieler 2 sich eine Botschaft ausdenkt, die unter keinen Umständen fehlinterpretiert werden kann, während das zweite, das sich letztendlich bewahrheitet, auf der Erwartung gründet, er werde denselben Fehler wie seine Vorgänger begehen. Zusammenfassend kann also behauptet

\footnotetext{
${ }^{9}$ Auch dem Leser der vorliegenden Arbeit sei diese Frage überlassen.

Pandaemonium, São Paulo, v. 21, n. 35, set.-dez. 2018, p. 47-68
} 
Stein, T. - Die audiovisuelle Übersetzung von Wortspielen

werden, dass alle Parameter, auf die der Übersetzer Einfluss hat, befolgt wurden und das Translat dem ursprünglichen Witz gemäß der GTVH sehr ähnlich ist.

Bedauerlicherweise überlassen bei Weitem nicht alle Wortspiele einen so großen Handlungsspielraum. Ein ausgezeichnetes Beispiel dafür ist Terapia de Angustia ${ }^{10}$ (s. Tabelle 2). In diesem Sketch beklagt sich Juan bei seinem Psychotherapeuten über etwas, das dieser als eine durch Angstzustände ausgelöste Schlafstörung versteht: „Doctor, la angustia no me deja dormir“" (Ich kann vor Angst nicht schlafen). Als Juan jedoch versucht einzuschlafen, tritt eine Frau namens Angustia ins Bild und weckt ihn gewaltsam auf.

TABELLE 2

Terapia de Angustia

\begin{tabular}{|c|c|c|c|}
\hline Zeitcode & Sprecher & Spanisch & Deutsch \\
\hline $\begin{array}{l}00: 03,680 \rightarrow \\
00: 05,840\end{array}$ & Therapeut & $\begin{array}{l}\text { Adelante, Juan. Tenemos una } \\
\text { hora. }\end{array}$ & $\begin{array}{l}\text { Na gut, Juan. Wir haben eine } \\
\text { Stunde. }\end{array}$ \\
\hline $\begin{array}{l}00: 06,200 \rightarrow \\
00: 08,980\end{array}$ & Juan & $\begin{array}{l}\text { Doctor, la angustia no me } \\
\text { deja dormir. }\end{array}$ & $\begin{array}{l}\text { Eine Frau raubt mir den } \\
\text { Schlaf. }\end{array}$ \\
\hline $\begin{array}{l}00: 10,300 \rightarrow \\
00: 11,290\end{array}$ & Therapeut & Angustia. & Liebeswahn. \\
\hline $\begin{array}{l}00: 12,120 \rightarrow \\
00: 15,160\end{array}$ & Therapeut & $\begin{array}{l}\text { Juan, recuerde que todos los } \\
\text { problemas tienen solución. }\end{array}$ & $\begin{array}{l}\text { Juan, jedes Problem hat eine } \\
\text { Lösung. }\end{array}$ \\
\hline $\begin{array}{l}00: 15,230 \rightarrow \\
00: 17,800\end{array}$ & Juan & $\begin{array}{l}\text { No, doctor, esto no tiene } \\
\text { solución. }\end{array}$ & Dieses nicht. \\
\hline $\begin{array}{l}00: 17,840 \rightarrow \\
00: 18,830\end{array}$ & Juan & Mire. & Schauen Sie. \\
\hline $\begin{array}{l}00: 20,960 \rightarrow \\
00: 21,950\end{array}$ & Angustia & ¡Oye, despierta! & Hey, aufwachen! \\
\hline $\begin{array}{l}00: 22,100 \rightarrow \\
00: 24,190\end{array}$ & Juan & $\begin{array}{l}\text { Ya, Angustia, ¡déjame } \\
\text { dormir! }\end{array}$ & $\begin{array}{l}\text { Lass mich doch endlich } \\
\text { schlafen! }\end{array}$ \\
\hline $\begin{array}{l}00: 24,270 \rightarrow \\
00: 25,240\end{array}$ & Therapeut & ¿Sí ve, doctor? & Sehen Sie? \\
\hline
\end{tabular}

Auch nach einer Analyse des Ausgangstextes stellt die soeben beschriebene Konstellation eine wahre Herausforderung dar. Das heißt, es kann zwar festgehalten werden,

\footnotetext{
${ }^{10}<$ https://www.youtube.com/watch?v=EFNkvKsgqas $>$. 
Stein, T. - Die audiovisuelle Übersetzung von Wortspielen

dass die komische Wirkung auf der Ambivalenz des Begriffs angustia ${ }^{11}$ beruht und das Ziel der Übersetzung somit darin besteht, dieses irreführende Spiel in der Zielsprache wiederzugeben, doch gibt es einige Aspekte, die dieses Vorhaben ungemein erschweren. Problematisch ist dabei in erster Linie die Tatsache, dass es in der deutschen Sprache keinen Frauennamen gibt, der als Ursache einer Schlafstörung gedeutet werden könnte. Gleichzeitig bleibt dem Übersetzer aufgrund des zu untertitelnden Videomaterials aber auch die Möglichkeit verwehrt, das unerwartete Auftreten einer Frau durch einen semantischen Baustein zu ersetzen, der sich im Deutschen besser für ein derartiges Wortspiel eignen würde.

Trotz dieser komplexen Situation konnte eine Lösung erarbeitet werden, die der perlokutionären Wirkung des Originals nahekommt. Dazu wurde gänzlich auf die Erwähnung von Angstzuständen verzichtet. Stattdessen kam der ambivalente Ausdruck jemandem den Schlaf rauben zum Einsatz und wurde sogleich mit einer Frau in Verbindung gebracht: „Eine Frau raubt mir den Schlaf“. ${ }^{12}$ Dieser Eingriff hatte zwangsläufig weitere Abweichungen vom Ausgangstext zur Folge. So notiert der Therapeut auf seinem Block „Angustia“, was in der Untertitelung zur Diagnose „Liebeswahn“ wurde.

Möchte man die Übersetzung nach der GTVH bewerten, fällt sofort auf, dass auch hier TA fehlt, während NS (Dialog) und SI (Therapiesitzung mit unerwartetem Besuch) bereits vorgegeben sind. LA entspricht in den Untertiteln „Eine Frau raubt mir den Schlaf“ und „Liebeswahn“ nicht dem Original, denn das durch die Situation (SI) vorgegebene Eingreifen der Figur Angustia begrenzt unwillkürlich die Möglichkeiten des sprachlichen Materials. LM betreffend schafft es die Übersetzung, die Auflösung des Sketches ebenfalls mittels eines cratylism herzustellen, indem der Rezipient durch die Zweideutigkeit des Ausdrucks jemandem den Schlaf rauben in die Irre geführt wird. Zu guter Letzt ist auch SO gegeben, auch wenn die gegenübergestellten Skripte ,Liebeswahn“ und ,Ruhestörerin“ denen des Ausgangstextes (,Angstzustände“ und ,Ruhestörerin') nicht ganz entsprechen. Somit kann geschlussfolgert werden, dass die Parameter LA und SO Abweichungen aufweisen. LA wird seiner Aufgabe, eine Auflösung des Witzes durch den vorgegebenen logischen Mechanismus zu gewährleisten,

\footnotetext{
${ }^{11}$ Ambivalent ist dieser Begriff ungeachtet des Gebrauchs des Definitartikels la vor angustia. In Ecuador ist es nichts Außergewöhnliches, dass in der Umgangssprache Personennamen ein Artikel vorangeht.

${ }^{12}$ Wie zu sehen ist, wurde die Anrede mit doctor in der Zielsprache nicht wiedergegeben. Das liegt nicht nur an der mit der Untertitelung einhergehenden Notwendigkeit der Kürzung, sondern auch daran, dass Vokative wie doctor und ingeniero im spanischsprachigen Raum oft auf keinen realen akademischen Titel verweisen, sondern als eine Art captatio benevolentiae gegenüber dem Angesprochenen verwendet werden. Näheres dazu findet sich in Stein (2018: 52).
} 
Stein, T. - Die audiovisuelle Übersetzung von Wortspielen

trotz allem gerecht. Da es sich bei SO jedoch um einen Parameter mit einer sehr hohen hierarchischen Stellung handelt, kann man im Sinne der GTVH behaupten, dass sich der Einsatz teils anderer - wenn auch sich überschneidender und entgegengesetzter - Skripte auf die Wahrnehmung des Witzes auswirkt. Zusammenfassend kann man also sagen, dass die angefertigte Untertitelung nicht in der Lage ist, die ursprüngliche Komik originalgetreu wiederzugeben. Angesichts der besonderen Bedingungen des hier behandelten Sketches wäre dies wohlgemerkt zumindest bei einer Übertragung in die deutsche Sprache, in der ein Wortspiel nach Vorbild des Ausgangstextes nicht in Frage kommt, nicht machbar gewesen.

Das letzte Beispiel, das hier behandelt wird, stammt aus Pollo al Vino (s. Tabelle 3). Unsere Auseinandersetzung mit dem audiovisuellen Übersetzen von Wortspielen findet in diesem Sketch ihren Höhepunkt, denn seine erfolgreiche Übertragung stellt eine derart große Herausforderung dar, dass die in der vorliegenden Arbeit vorgestellten Methoden einem wahren Härtetest unterzogen werden. In Pollo al Vino begegnet man einem Paar bei einem romantischen Abendessen im Restaurant. Bei einem Gespräch über ausgefallene Speisen fragt der Mann seine Begleiterin plötzlich: „¿Te gusta el... pollo al vino?“ (Isst du gerne Coq au Vin?). Als sie das bejaht, dreht er sich zu einem dritten Tischgenossen um, der bisher nicht im Bild war und Albino ist, und sagt: „Listo, Pollo, hoy tiras“ (Alles klar, Pollo, heute hast du Sex).

TABELLE 3

Pollo al Vino

\begin{tabular}{|c|c|c|c|}
\hline Zeitcode & Sprecher & Spanisch & Deutsch \\
\hline $\begin{array}{l}00: 03,680 \rightarrow \\
00: 05,340\end{array}$ & Mann & $\begin{array}{l}\text {...y encima le echo un poco } \\
\text { de pimienta con los dedos. }\end{array}$ & $\begin{array}{l}\text {...und nur noch eine Prise } \\
\text { Pfeffer. }\end{array}$ \\
\hline $\begin{array}{l}00: 05,420 \rightarrow \\
00: 07,150\end{array}$ & Frau, Mann & $\begin{array}{l}\text {-Con salsa tártara. } \\
\text {-¡Exacto! }\end{array}$ & $\begin{array}{l}\text {-Mit Sauce tartare. } \\
\text {-Genau! }\end{array}$ \\
\hline $\begin{array}{l}00: 07,190 \rightarrow \\
00: 09,440\end{array}$ & Frau, Mann & $\begin{array}{l}\text {-¡Es delicioso! } \\
\text {-Uno de mis favoritos. }\end{array}$ & $\begin{array}{l}\text {-Köstlich! } \\
\text {-Eines meiner Leibgerichte. }\end{array}$ \\
\hline $\begin{array}{l}00: 09,480 \rightarrow \\
00: 10,620\end{array}$ & Frau & Ya somos dos. & Da sind wir schon zwei. \\
\hline $\begin{array}{l}00: 11,400 \rightarrow \\
00: 12,860\end{array}$ & Mann & ¿Y qué más te gusta? & Was naschst du noch gern? \\
\hline $\begin{array}{l}00: 12,900 \rightarrow \\
00: 14,700\end{array}$ & Frau, Mann & $\begin{array}{l}\text {-No sé. } \\
\text {-Debe haber algo. }\end{array}$ & $\begin{array}{l}\text {-Weiß nicht. } \\
\text {-Etwas Exotisches? }\end{array}$ \\
\hline $\begin{array}{l}00: 15,320 \rightarrow \\
00: 16,370\end{array}$ & Frau & Dame una opción. & Was zum Beispiel? \\
\hline $\begin{array}{l}00: 16,880 \rightarrow \\
00: 18,260\end{array}$ & Mann & ¿Te gusta el... & Wie wäre es mit einem... \\
\hline
\end{tabular}


Stein, T. - Die audiovisuelle Übersetzung von Wortspielen

\begin{tabular}{llll}
$00: 19,380 \rightarrow$ & Mann & pollo al vino? & Albino-Hecht \\
$00: 20,410$ & & & \\
\hline $00: 21,280 \rightarrow$ & Frau & ¡Me encanta! & Liebend gern! \\
$00: 22,060$ & & & \\
\hline $00: 22,080 \rightarrow$ & Mann, & -Listo, Pollo, hoy tiras. & -Heute wirst du vernascht. \\
$00: 24,120$ & Albino & -Ya era horaf ${ }^{13}$. & -Na endlich.
\end{tabular}

Das Wortspiel, das diesem Dialog zugrunde liegt, ist äußerst subtil und bedarf einer genauen Erläuterung. Zum einen bedeutet pollo zwar grundsätzlich Hähnchen, kann in Ecuador jedoch als freundschaftlicher Spitzname für Männer gebraucht werden; und zum anderen gibt es keinen vernehmbaren phonetischen Unterschied zwischen den Ausdrücken al vino und albino, ihre Aussprache ist identisch: [al'ßino]. Daraus ergibt sich für den Zieltext die Notwendigkeit einer Überleitung vom Gericht auf den unerwarteten Tischgenossen, die sich eines ambivalenten und zwei unterschiedliche Skripte zulassenden Begriffs bedient.

Es steht fest, dass das Kernstück der zielsprachlichen Wiedergabe erneut die Täuschung des Rezipienten sein muss. Dabei kann man nicht einfach über das plötzliche Auftreten eines Mannes mit Albinismus hinwegsehen. Die Bedingungen werden zusätzlich dadurch erschwert, dass das französische Nationalgericht pollo al vino im deutschsprachigen Raum als Coq au Vin bekannt ist. Da die Suche nach einem homophonen deutschen Begriff aus diesem Grund zum Scheitern verurteilt ist, bleibt entsprechend Hickeys (1999) Ansätzen als einzige Alternative nur noch die Ausarbeitung eines Parallelwitzes. Um das Skript ,Gespräch über ausgefallene Speisen` zu bewahren, wurde dazu das Polysem Hecht herangezogen, denn dieses Nomen kann einerseits einen Fisch und andererseits einen bewundernswerten Mann bezeichnen (SCHOLZESTUBENRECHT 2015). Insgesamt war eine tiefgreifende Umgestaltung des zielsprachlichen Witzes notwendig. So wurde gustar aus „¿Y qué más te gusta?“ (Was isst du noch gerne?) als naschen übersetzt: „Was naschst du noch gern?“ Dadurch sollte die Zweideutigkeit dieses Verbs, das sowohl auf sexuelle Anziehung als auch auf kulinarische Vorlieben verweisen kann, erhalten bleiben. Gleichzeitig gelingt damit aber auch die Überleitung auf den erotischen Kommentar am Ende des Sketches: „Heute wirst du vernascht“. Für eine kohärente Gestaltung des zielsprachlichen Witzes waren darüber hinaus weitere Umstrukturierungen erforderlich. So ist von einem Albino-Hecht die Rede, da das Auftauchen eines Mannes mit Albinismus sonst

\footnotetext{
13 Die scheinbar fehlerhafte Schreibweise von horaf war beabsichtigt, denn sie spiegelt den von der Figur gesprochenen Dialekt Quitos wider, in dem an das letzte Wort eines Satzes oft -f angehängt wird: „¿Cuál es la única ciudad del Ecuador cuyo nombre termina en f?: ¡Quitof!“‘ (RODRÍGUEZ 2016).
} 
Stein, T. - Die audiovisuelle Übersetzung von Wortspielen

ungeklärt bliebe. Da nur sehr wenige dieser Fische Albinismus haben (WÜSTNECK 2016), wurde ergänzt, es handele sich um ein exotisches Gericht: „Etwas Exotisches?“ Was schließlich die nominale Anrede in „Listo, Pollo, hoy tiras“ anbelangt, verzichtet der zielsprachliche Text auf den Versuch, sie einzubeziehen, da sie für die Herstellung des Wortspiels in deutscher Sprache nicht benötigt wird.

Wie soeben zu sehen war, bedurfte es vieler Eingriffe, um die perlokutionäre Wirkung des Sketches, die auf einem Wortspiel aufbaut, wiederzugeben. Nun gilt es zu erörtern, ob das Ergebnis als zufriedenstellend gewertet werden kann. Wie immer seien zuerst diejenigen Parameter genannt, auf die der Übersetzer keinen Einfluss hat. Dazu zählen abermals NS, TA und SI. TA liegt aufgrund des Fehlens superioritätsbasierter Komik nicht vor. Sowohl die erzählerische Struktur als auch die Situierung sind im audiovisuellen Material inbegriffen. Wie bereits zu sehen war, wirkt sich letztere insbesondere durch das unerwartete Auftreten einer dritten Figur stark einschränkend auf den Handlungsspielraum des Übersetzers aus. Das äußert sich in erster Linie an LA, was zu einer Art Kettenreaktion führt. So musste das sprachliche Material vielerlei Änderungen unterzogen werden, die wiederum LM betreffen, da die Auflösung des Wortspiels nicht mehr nach Vorbild des Originals vonstattengehen kann: Zwar kommt immer noch der logische Mechanismus cratylism zur Anwendung, jedoch entspringt er der Polysemie des Begriffs Hecht und nicht der Homophonie zweier unterschiedlicher Ausdrücke, wie es bei al vino und albino der Fall ist. Zu guter Letzt kann in Bezug auf SO festgestellt werden, dass die Opposition und Überschneidung der Skripte ,Gespräch über ausgefallene Speisen` und ,Gespräch über einen Mann mit Albinismus' erhalten bleibt. Das bedeutet in erster Linie, dass auch die Übersetzung vom Rezipienten als belustigend aufgefasst werden dürfte. Allerdings ist meines Erachtens deutlich zu sehen, dass es der hergestellten Skriptopposition an der Natürlichkeit des Ausgangstextes fehlt. Insbesondere der Einbezug des Albino-Hechts, der nicht gerade an ein konventionelles Gericht erinnert, dürfte bei der Rezeption der Untertitelung wohl als befremdend empfunden werden, was bei pollo al vino nicht der Fall ist. Zusammenfassend kann also gesagt werden, dass sich bei der Anwendung der GTVH zwar nur nennenswerte Abweichungen in LA erkennen lassen, diese sich jedoch auf die anderen Parameter auswirken, was zu einem recht forcierten Gesamteindruck führt. 


\section{Abschließende Bemerkungen}

Die in der vorliegenden Arbeit vorgestellten Übersetzungs- und Evaluierungsstrategien dienten als Ausgangspunkt für eine translationsbezogene Auseinandersetzung mit den betreffenden Sketchen. Die komische Wirkung in einer anderen Sprache zu reproduzieren und damit nachvollziehbar zu machen, ist keine leichte Aufgabe. Um sie zu bewältigen, gibt Hickey (1999) dem Translator eine zwar simple, doch deswegen nicht minder nützliche Methode an die Hand. Die Idee, die Essenz der Komik herauszufiltern und sie erst dann in eine andere Sprache und/oder Kultur zu transferieren, bildet einen äußerst praxisnahen Ansatz. Auch wenn die meisten Übersetzer diesen Weg intuitiv einschlagen würden, kann eine solche methodische Untermauerung durchaus als ein wichtiger Anstoß zur Reflexion angesehen werden.

Die GTVH ermöglicht ein Urteil darüber, ob die Übersetzung eines humoristischen Textes als zufriedenstellend zu bezeichnen ist. Das entscheidende Kriterium ist dabei eine an das Original angelehnte Wiedergabe auf den unterschiedlichen Ebenen, aus denen sich ein Witz zusammensetzt. Auch wenn Attardo (2002) einräumt, dass eine Beachtung aller Parameter bei Weitem nicht immer möglich noch erforderlich ist, ${ }^{14}$ nimmt das Original dadurch eine höhere Stellung ein, als es bei Hickey (1999) der Fall ist. Da es für die Übertragung eines jeden Wortspiels jedoch einer ganz eigenen Vorgehensweise bedarf, können meines Erachtens keine allgemeingültigen Aussagen in Bezug auf die Bedeutung des Originals für den Translationsprozess getroffen werden. So dürfte oftmals lediglich die Distanzierung vom ausgangssprachlichen Wortspiel zu einem akzeptablen Ergebnis in der Zielsprache führen, weshalb es letztlich dem Übersetzer überlassen sein sollte, seine Prioritäten je nach Situation eigenständig zu setzen.

Die Limitationen der hier besprochenen Methoden wurden am Beispiel von Terapia de Angustia und Pollo al Vino sehr deutlich, denn sie sind für das audiovisuelle Übersetzen nicht ausgelegt. Die Bindung an die Zeichenmodalität ,Bild“ machte hier ein großes Maß an

\footnotetext{
${ }^{14}$ In diesem Sinne befürwortet Attardo (2002) den Austausch bestimmter Parameter, falls sie nicht zum Erzielen komischer Wirkung in der Zielsprache geeignet sind, so beispielsweise SI: „If the translator should find $\mathrm{him} / \mathrm{her} /$ itself in a situation in which the Situation is either non-existent in the TL or else unavailable for humour, a good solution is simply to replace the offending Situation with another one, while respecting all other Knowledge Resources"(187 f.).
} 
Stein, T. - Die audiovisuelle Übersetzung von Wortspielen

Kreativität seitens des Übersetzers erforderlich. ${ }^{15}$ Dieser Umstand zeugt von der Notwendigkeit, die bestehenden Methoden zur Übersetzung des Komischen auf die besonderen

Bedürfnisse der Untertitelung auszuweiten. Ein solcher Beitrag zur Professionalisierung der Sprachmittler wäre angesichts der voranschreitenden Internationalisierung audiovisueller Medien humoristischen Inhalts sicherlich willkommen.

\section{Literaturverzeichnis}

Attardo, Salvatore; HemPelmann, Christian; MaIO, Sara Di. Script oppositions and logical mechanisms: modeling incongruities and their resolutions. Humor, v. 15, n. 1, p. 3-46, 2002.

ATTARDO, Salvatore. Translation and humour: an approach based on the General Theory of Verbal Humour (GTVH). The Translator, v. 8, n. 2, p. 173-194, 2002.

ATTARDO, Salvatore; RASKIN, Victor. Script theory revis(it)ed: joke similarity and joke representation model. Humor, v. 4, n. 3-4, p. 293-347, 1991.

DelaBASTITA, Dirk. Introduction. The Translator, v. 2, n. 2, p. 127-139, 1996.

DIOT, Roland. Humor for intellectuals: can it be exported and translated? Meta, v. 34, n. 1, p. 84-87, 1989.

HICKEY, Leo. Aproximación pragmalingüística a la traducción del humor. In: GIL DE CARRASCO, Antonio; HiCKEY, Leo (Org.). 1999 - Aproximaciones a la traducción. Disponível em: <http://cvc.cervantes.es/lengua/aproximaciones/hickey.htm> Acesso em: 27 maio 2018.

HobBeS, Thomas. Vom Menschen. Hamburg: Meiner, 1966.

KANT, Immanuel. Critik der Urtheilskraft. Frankfurt, 1792.

REISS, Katharina. Grundfragen der Übersetzungswissenschaft. Wiener Vorlesungen. HermesWUV, 2000.

RodRíGUEZ, Mario. La jerga "made in Ecuador". El Telegrafo, Paysandú 28.08.2016. Disponível em: $<$ http://www.eltelegrafo.com.ec/noticias/septimo-dia/51/la-jerga-made-in-ecuador>. Acesso em: 18 jul. 2017.

SANTANA LÓPEZ, Belén. Wie wird das Komische übersetzt? Berlin: Frank \& Timme, 2006.

SCHOLZE-STUBENRECHT, Werner (Org.). Duden, Deutsches Universalwörterbuch: Das umfassende Bedeutungswörterbuch der deutschen Gegenwartssprache. Berlin: Dudenverlag, 2015. 2128 p.

SCHOPENHAUER, Arthur. Arthur Schopenhauers sämmtliche Werke: Die Welt als Wille und Vorstellung. Leipzig: Brockhaus, 1916. (2).

SCHROEDER, Susanne. "Lachen ist gesund?" - eine volkstümliche und medizinische Binsenwahrheit im Spiegel der Philosophie. Tese de doutorado. Freie Univerisität Berlin, Berlim, 2002.

STACKELBERG, Jürgen von. Translating comical writing. Translation Review, v. 28, n. 1, p. 10-14, 1988.

STEIN, Timur. Das Komische und seine Untertitelung: Herausforderungen und Lösungsansätze am Beispiel der ecuadorianischen Webserie Enchufe.tv, Johannes Gutenberg-Universität Mainz, Mainz, 2018.

VANDAELE, Jeroen. Humor mechanisms in film comedy: incongruity and superiority. Poetics Today, v. 23, n. 2, p. 221-249, 2002.

\footnotetext{
${ }^{15}$ Für weitere Beispiele dafür, wie sich die unterschiedlichen Zeichenmodalitäten auf den Übersetzungsprozess auswirken können, siehe Stein (2018: 55 ff.)
}

Pandaemonium, São Paulo, v. 21, n. 35, set.-dez. 2018, p. 47-68 
Stein, T. - Die audiovisuelle Übersetzung von Wortspielen

VANDAELE, Jeroen. Humor in translation. In: GAMBIER, Yves; VAN DOORSLAER, Luc (Org.). Handbook of Translation Studies. Amsterdam: John Benjamins, 2010 p. 147-152.

WÜSTNECK, Bernd. Müritzeum verliert Aushängeschild: Goldener Hecht gestorben. Focus Online, Munique, 10.11.2016. Disponível em: <http://www.focus.de/regional/mecklenburgvorpommern/natur-mueritzeum-verliert-aushaengeschild-goldener-hechtgestorben_id_6188483.html>. Acesso em: 22 maio 2018.

Recebido em 15 de março de 2018 Aceito em 28 de abril de 2018 